\title{
TRANSONIC HYDRODYNAMIC ESCAPE OF HYDROGEN FROM EXTRASOLAR PLANETARY ATMOSPHERES
}

\author{
Feng Tian, ${ }^{1,2}$ Owen B. Toon, ${ }^{2,3}$ Alexander A. Pavlov, ${ }^{2}$ and H. De Sterck ${ }^{4}$ \\ Received 2004 May 18; accepted 2004 October 28
}

\begin{abstract}
Hydrodynamic escape is an important process in the formation and evolution of planetary atmospheres. Transonic steady state solutions of the time-independent hydrodynamic equations are difficult to find because of the existence of a singularity point. A numerical model is developed to study the hydrodynamic escape of neutral gas from planetary atmospheres by solving the time-dependent hydrodynamic equations. The model is validated against an analytical solution of the escape from an isothermal atmosphere. The model uses a two-dimensional energy deposition calculation instead of the single-layer heating assumption, which is not sufficiently accurate for hydrodynamic escape from a hydrogen-rich planetary atmosphere. When applied to the atmospheres of extrasolar planets, the model results are in good agreement with observations of the transiting extrasolar planet HD 209458b. The model predicts that hydrogen is escaping from HD 209458b at a maximum rate of $6 \times 10^{10} \mathrm{~g} \mathrm{~s}^{-1}$. The extrasolar planet is stable under the hydrodynamic escape of hydrogen. The rate of hydrogen hydrodynamic escape from other possible extrasolar planets is investigated using the model. The importance of hydrogen hydrodynamic escape for the long-term evolution of extrasolar planets is discussed. Simulation shows that through hydrodynamic escape of hydrogen, a planet at the orbit of Mercury $(0.4 \mathrm{AU})$ and with 0.5 Uranus mass can lose about $10 \%$ of its mass within 850 million yr if the solar EUV radiation is 10 times the present level. This calculation provides an indication of how Mercury may have evolved during the early days of the solar system.
\end{abstract}

Subject headings: planetary systems — planets and satellites: general

\section{INTRODUCTION}

Typically, the process of atmospheric gas loss to space involves a static background heavy gas and a small amount of "light" escaping gas. "Light" particles in the upper atmosphere moving in the outward direction with a velocity large enough to overcome the gravitational trap will escape from the planet (Shizgal \& Arkos 1996). However, in an atmosphere experiencing hydrodynamic escape, the background gas cannot be considered as static. In this case, the behavior of the upper atmosphere can be described by fluid equations. Hydrodynamic escape is one of a few mechanisms that can change the composition of a planetary atmosphere irreversibly (Hunten 1990). Venus may have lost an ocean of water by hydrodynamic escape of hydrogen (Kasting \& Pollack 1983). Hydrodynamic escape can also help to explain why some isotopic ratios $(\mathrm{D} / \mathrm{H}, \mathrm{N}$, and noble gases) are so different on terrestrial planets, even though these planets formed from similar material (Hunten et al. 1987; Pepin 1991). Because of its weak gravity field, Pluto's atmosphere may experience hydrodynamic escape of $\mathrm{N}_{2}$ at present (Krasnopolsky 1999). The Earth is believed to have had an anoxic atmosphere 2.45 billion yr ago (Pavlov \& Kasting 2002). Without atomic oxygen, the exobase might have been cold enough that thermal escape was inefficient compared with hydrodynamic escape. In that case, the hydrogen abundance in the atmosphere would be controlled by the hydrodynamic escape of hydrogen. Hydrodynamic escape may also be an ongoing process on some extrasolar planets (Vidal-Madjar

\footnotetext{
${ }^{1}$ Astrophysical and Planetary Science Department, University of Colorado, UCB 391, Boulder, CO 80309.

${ }_{2}$ Laboratory for Atmospheric and Space Physics, University of Colorado, 1234 Innovation Drive, Boulder, CO 80309.

${ }_{3}$ Program in Atmospheric and Oceanic Science, University of Colorado, UCB 311, Boulder, CO 80309.

${ }^{4}$ Department of Applied Mathematics, University of Waterloo, Waterloo, ON N2L 3G1, Canada.
}

et al. 2003, 2004), and its impact on the evolution of small-orbit extrasolar planets can be significant if enough energy is deposited in the planetary atmosphere.

Several numerical models have been developed previously to solve the hydrodynamic escape problem (Watson et al. 1981; Kasting \& Pollack 1983; Chassefière 1996). Because a singularity point (where the gas flow velocity equals the sound speed) exists in the transonic time-independent hydrodynamic equations, a self-consistent transonic solution of the time-independent equations cannot be obtained easily. Watson et al. (1981) used a trial-and-error method to solve the problem. For a certain value of escape flux, this method calculates the required density and velocity distribution in the steady state. A $10 \%$ variation of the mass flux at the critical point can cause a change of density and velocity at the lower boundary by a factor of 10 or more (sometimes more than 4 orders of magnitude). Therefore, it is difficult to find solutions for a pure hydrogen atmosphere using this method (Kasting \& Pollack 1983). Watson et al. assumed that heating by solar EUV radiation occurred in a single layer. Because the optical depth depends on the absorption cross section, the altitude of unity optical depth is not the same for solar radiation at different wavelengths. In fact, the heating rate distribution has a broad structure (Fox \& Dalgarno 1979; Chassefière 1996). The impact of Watson's single-layer heating assumption is discussed in this paper. Kasting \& Pollack (1983) included photochemistry as well as infrared cooling by $\mathrm{H}_{2} \mathrm{O}$ and $\mathrm{CO}_{2}$ in a study of the hydrodynamic escape from the atmosphere of early Venus. They developed a numerical method to solve the momentum and the energy equations iteratively. Their method was limited to subsonic solutions. Chassefière (1996) matched the hydrodynamic escape flux to a modified Jeans escape flux at the exobase level and considered the interaction between the solar wind and the early Venusian atmosphere. This model forbids transonic solutions, because the exobase level is set to be below the transonic level. The single-layer heating assumption and the 
TABLE 1

Constraints on the Boundary Conditions for Transonic Hydrodynamic Escape

\begin{tabular}{|c|c|c|}
\hline Problem & Lower Boundary & Upper Boundary \\
\hline 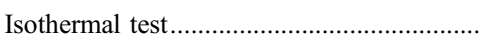 & 1 variable to be imposed, 1 variable to be extrapolated & 2 variables to be extrapolated (no boundary conditions) \\
\hline Full set of hydrodynamic equations ............ & 2 variables to be imposed, 1 variable to be extrapolated & 3 variables to be extrapolated \\
\hline
\end{tabular}

subsonic approximation limit the possibility of a self-consistent study of the hydrodynamic escape of neutral gases from planetary atmospheres. A robust numerical scheme capable of solving the transonic hydrodynamic escape problem with a distributed energy source is needed.

Here we present the first study on the time-dependent hydrodynamic equations for transonic neutral gas escape from planetary atmospheres. The singular behavior in the time-independent hydrodynamic equations no longer exists in the time-dependent model, thus allowing the transonic solution to be obtained through time marching to a steady state. Our dynamic model is onedimensional, since a spherically symmetric atmosphere is assumed. Because of the unsymmetrical nature of the external energy input in planetary atmospheres, a separate two-dimensional model is necessary for the energy deposition calculation (details of this model in $\S 3$ ). The combination of a one-dimensional dynamic model with the two-dimensional energy deposition model will provide a good first-order approximation of the real hydrodynamic escape process from planetary atmospheres.

The solution technique and numerical methods are introduced in $\S 2$. In the same section we validate our solution technique against analytical solutions for an isothermal atmosphere. Implications of the single-layer heating assumption along with the two-dimensional energy deposition model and a discussion of the heating efficiency are included in $\S 3$. In $\S 4$ the model is applied to a hydrogen-dominated atmosphere of an Earth-like planet (with mass, radius, and solar EUV flux level the same as those of the Earth). The results are compared with the Watson et al. (1981) simulation results for a similar atmosphere. Important features of the hydrodynamic escape flow are discussed. We apply the model to the extended atmosphere of extrasolar planet HD 209458b in $\S 5$ and try to explain recent Ly $\alpha$ observations (Vidal-Madjar et al. 2003) and oxygen and carbon observations (Vidal-Madjar et al. 2004). We also present simulations of hydrogen hydrodynamic escape from small-orbit extrasolar planets with different parameters in $\S 5$.

\section{DESCRIPTION OF SOLUTION TECHNIQUE}

The one-dimensional time-dependent nonviscous hydrodynamic equations for a single-constituent atmosphere with thermal conduction in spherical geometry are

$$
\begin{aligned}
\frac{\partial\left(\rho r^{2}\right)}{\partial t}+\frac{\partial\left(\rho u r^{2}\right)}{\partial r} & =0 \\
\frac{\partial\left(\rho u r^{2}\right)}{\partial t}+\frac{\partial\left(\rho u^{2} r^{2}+p r^{2}\right)}{\partial r} & =-\rho G M+2 p r \\
\frac{\partial\left(E r^{2}\right)}{\partial t}+\frac{\partial\left[(E+p) u r^{2}\right]}{\partial r} & =-\rho u G M+q r^{2}+\frac{\partial}{\partial r}\left(\kappa r^{2} \frac{\partial T}{\partial r}\right),
\end{aligned}
$$

where $E=\rho\left(u^{2} / 2+e\right), e=p /[\rho(\gamma-1)]$, and $p=\rho R T$.

Here $\rho$ is the gas density, $r$ is the distance from the planet center, $u$ is the gas flow velocity, $p$ is the pressure, $G$ is the universal gravitational constant, $M$ is the mass of the planet, $E$ is the total energy density (which is the sum of the kinetic energy density and the internal energy density of the gas flow), $\kappa$ is the thermal conductivity, $T$ is the temperature, $\gamma$ is the adiabatic constant, $R$ is the molar gas constant, and $q$ is the volume heating rate (more details of heating rate are given in $\S 3$ ). Here $\rho u r^{2}$ is a measure of the globally integrated escape rate and must be a constant in space in the steady state. The $2 p r$ term on the right-hand side of the momentum equation is a part of the pressure gradient, so the homogeneous part of the equations (left-hand side) can be described as a hyperbolic system. The Lax-Friedrichs (LF) scheme (Toro 1999; De Sterck et al. 2001) is used to solve the system. The LF scheme includes an embedded explicit diffusion term that helps to stabilize the system under the Courant-Friedrichs-Lewy (CFL) condition ( Toro 1999; De Sterck 1999). When using an explicit time-marching method, the maximum time step is constrained by the CFL condition, which is dependent on the size of the grid cells. The distribution of grid cells in the model is not uniform. The grid size decreases exponentially with decreasing $r$ to compensate for the nearly exponential increase of density near the lower boundary (Keppens \& Goedbloed 1999). Local time steps can be defined by applying the CFL condition to each grid cell separately. A global time step is then determined as the minimum of the local time steps. The global time step is unnecessarily restrictive in large parts of the model. Using local time steps for different grid cells often turns out to be a more efficient time-marching method.

When applying the LF scheme to a hyperbolic system, the number of boundary conditions required depends on the relationship at the boundaries between the gas flow velocity and the sound speed in the system. If the gas flow velocity is greater than the sound speed, the flow is supersonic. In the case of hydrodynamic escape, the flow is slow at the lower boundary (subsonic) and fast at the upper boundary (usually supersonic). Table 1 summarizes the theoretical constraints on the number of boundary condition settings at both boundaries. In the numerical model, two ghost cells each are located below the lower and above the upper boundaries. Boundary conditions are imposed in the ghost cells, or the variables are extrapolated linearly to the ghost cells if there is no boundary condition to be imposed.

The steady state results are insensitive to the initial conditions. The initial conditions in our numerical model are set so that density is proportional to $r^{-2}$, and velocity is set to be a small constant for all grid cells. Convergence to a steady state is measured by the normalized difference of all variables between time steps,

$$
\Delta_{2} U=\sqrt{\frac{1}{N_{\text {var }}} \sum_{u=1}^{N_{\text {var }}}\left[\frac{1}{N_{\text {grid }}} \sum_{\text {grid }=1}^{N_{\text {grid }}} \frac{\left(U_{u}^{n+1}-U_{u}^{n}\right)^{2}}{\left(U_{u}^{n}\right)^{2}}\right]} .
$$

Here $n$ represents the time level, $N_{\text {var }}$ is the number of variables in the system $\left(N_{\text {var }}=2\right.$ in the isothermal hydrodynamic system), and $N_{\text {grid }}$ is the number of grid cells in the system. In the model the steady state tolerance is set at $\Delta_{2} U=10^{-10}$. Sensitivity tests show that if the steady state tolerance is set to 

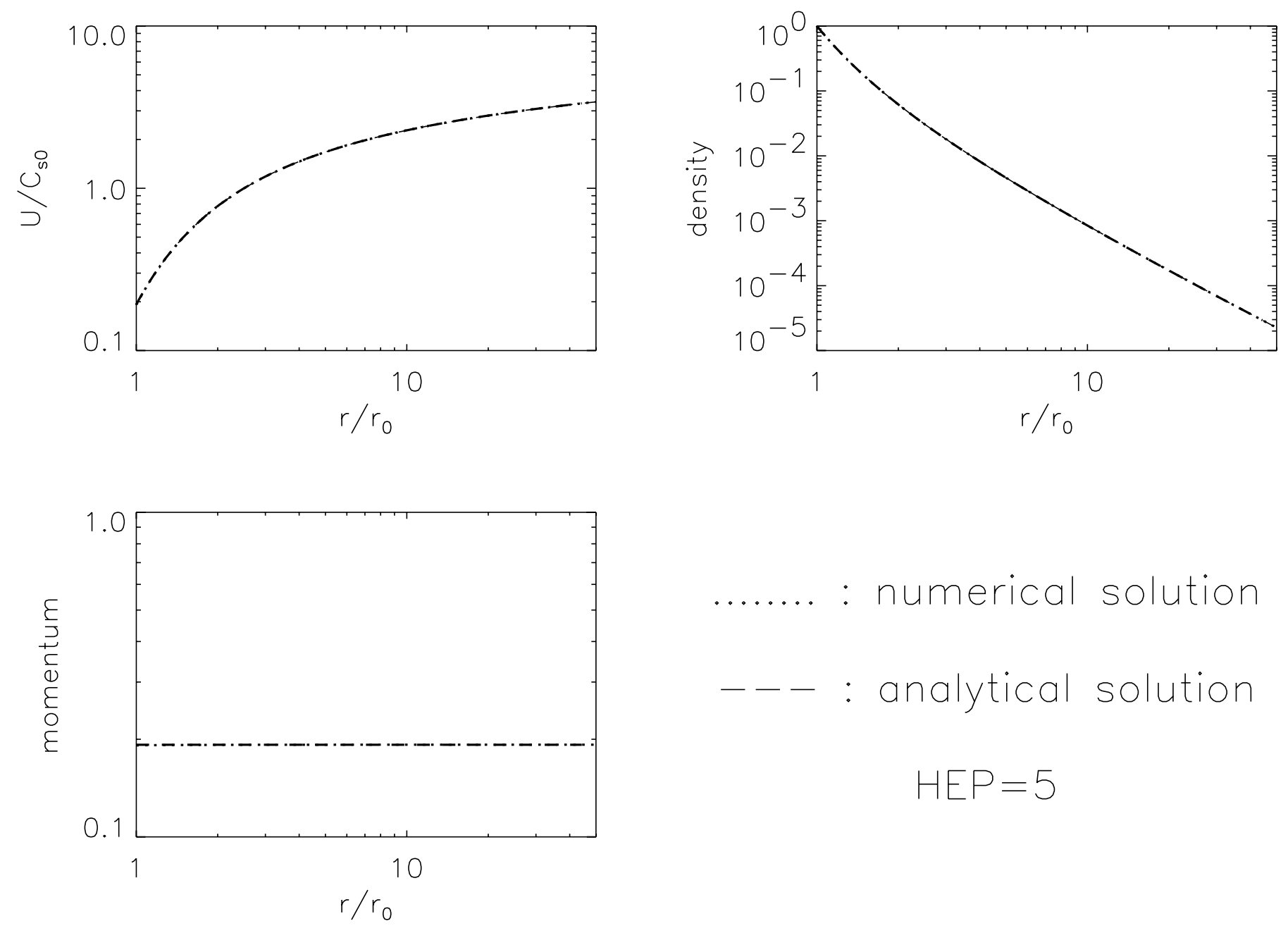

FIG. 1.-Comparison between the numerical solution and the analytical solution for the HEP $=5$ case.

a value that is not small enough, e.g., $10^{-8}$, the steady state solution may not be approached sufficiently.

\subsection{Validation in an Isothermal Atmosphere}

Here we validate our solution technique against analytical solutions for an isothermal atmosphere. Similar validation tests can be found in Keppens \& Goedbloed (1999). In an isothermal atmosphere, equation (1) can be reduced to the density and the momentum equations. An analytical solution with a nonzero velocity distribution is available (Parker 1964). In our numerical model, the density is imposed, and the mass flux is extrapolated at the lower boundary.

The ratio of the gravitational potential energy and the molecular kinetic energy is important to identify the dynamic characteristics of the escape. The traditional Jeans escape parameter is defined by parameters at the exobase level. In an atmosphere in which hydrodynamic escape occurs, the location of the exobase is unknown prior to the simulations, so we define the hydrodynamic escape parameter (HEP) using the parameters at the lower boundary level: HEP $=G M m / r_{0} k T_{0}$, where $m$ is the particle mass, $k$ is Boltzmann's constant, and $r_{0}$ and $T_{0}$ are the values of $r$ and $T$ in equation (1) at the lower boundary, respectively. When the kinetic energy of molecules is comparable to their gravitational potential energy, the HEP value is small and the escape flow is strong (e.g., HEP $=5$ in Fig. 1). When the gravitational energy is much larger than the kinetic energy, the HEP is large and the escape flow is weak (e.g., HEP $=15$ in Fig. 2). The scaled mass flux (momentum) in the HEP $=15$ case is more than 2 orders of magnitude smaller than that in the $\mathrm{HEP}=5$ case. Different HEP values also result in different density gradients. When $r$ changes from 1 to 2 , density drops by almost 4 orders of magnitude in the HEP $=15$ case but only by 2 orders of magnitude in the HEP $=5$ case.

Large number ranges and steep gradients pose a difficult challenge to numerical approximation techniques, and it can generally be expected that in regions of large gradients numerical accuracy may decrease. In the first-order LF scheme, an explicit diffusion term is included. This diffusion acts to damp sharp gradients on the grid, providing numerical stability. It is proportional to $\Delta x\left(\partial^{2} u / \partial x^{2}\right)$, where $\Delta x$ is the grid size and $\partial^{2} u / \partial x^{2}$ is the second derivative of the variables ( $u$ is density in the continuity equation). The constant of proportionality is the average of the wind speed and the speed of sound. Because of the large density curvature near the lower boundary (especially for the large-HEP case), the diffusion term is not negligible there. In the steady state, the time derivative term in the continuity equation goes to zero. If the explicit diffusion term is small, a nearly constant momentum can be obtained (analytical solution and HEP $=5$ ). However, near the lower boundary for large HEP the momentum must adjust itself to meet the large density curvature in the diffusion term, and thus it deviates from the analytical solution. As a result, in our numerical results the 

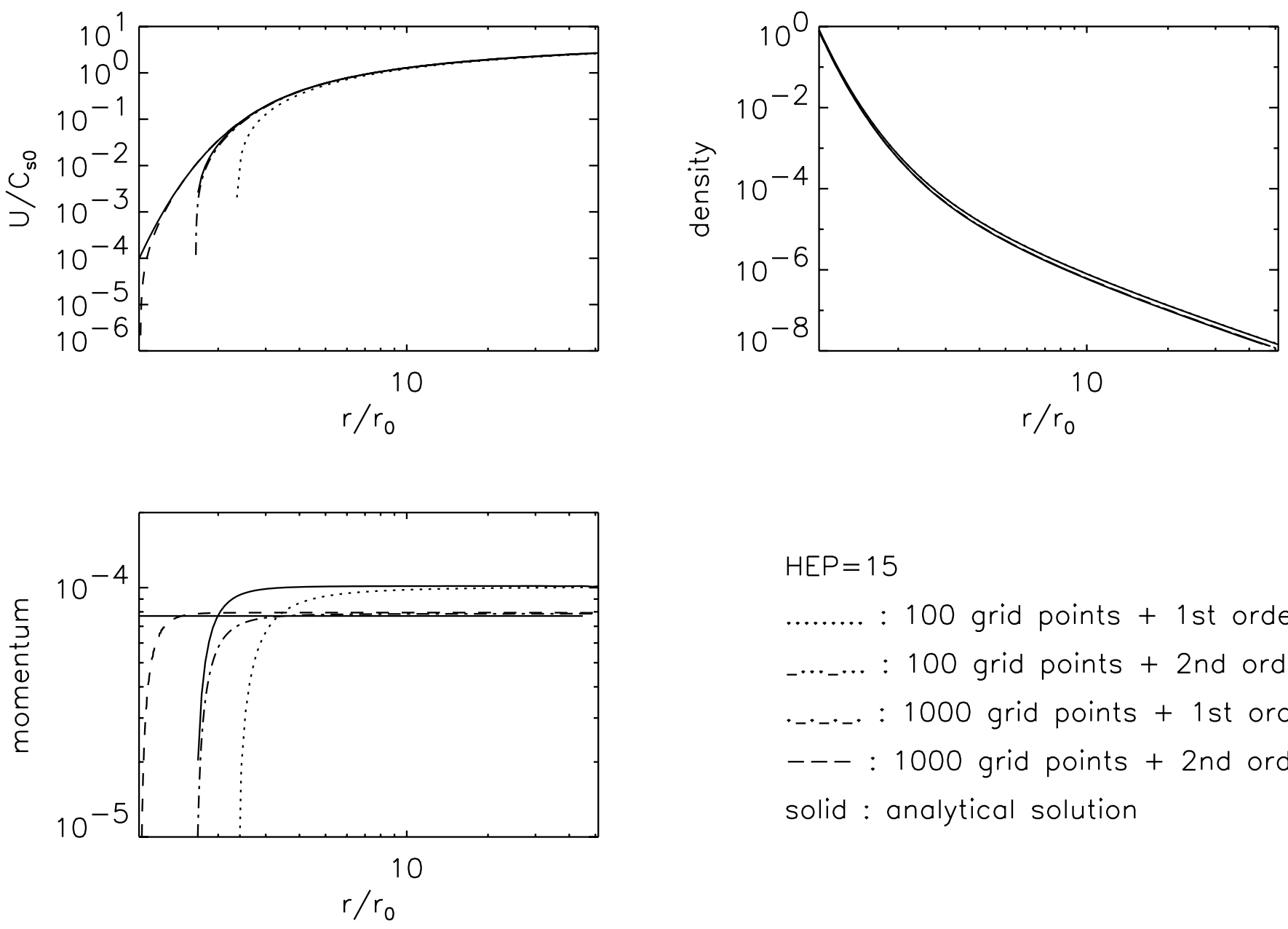

$$
\begin{aligned}
& \mathrm{HEP}=15 \\
& \text {....... : } 100 \text { grid points }+1 \text { st order } \\
& \text { _......: } 100 \text { grid points + 2nd order } \\
& \text {...... : } 1000 \text { grid points + 1st order } \\
& ---: 1000 \text { grid points }+ \text { 2nd order } \\
& \text { solid : analytical solution }
\end{aligned}
$$

FIG. 2.-Comparison between the numerical solutions and the analytical solution for the HEP $=15$ case.

momentum and the flow velocity are not calculated as accurately in the region of the domain close to the lower boundary as in the rest of the domain.

As illustrated in Figure 2, the accuracy in the region close to the lower boundary can be improved by refining the grid (globally or, preferentially, in the high-gradient region) or by increasing the order of the numerical approximation from first to second order (De Sterck et al. 2001). The fact that the numerical solution converges to the analytical solution as the grid is refined or the numerical approximation order is increased (Fig. 2) shows that the equations and boundary conditions are implemented correctly. It is also interesting to note that the deviation in momentum close to the lower boundary at low resolution does not influence the calculated value of the mass flux at the outflow boundary significantly. This is because the diffusion term becomes very small in most of the atmosphere, since the gradients are low. The resolution in the simulations to be presented below has been chosen to ensure that the calculated outflow mass flux is accurate and does not change substantially upon further refining the grid.

Figure 3 shows that for different HEP values, the analytical solutions are approached increasingly well by the numerical solutions when the number of grid cells is increased (first-order scheme). In the case of hydrogen-dominated atmospheres of the Earth and extrasolar planets, the simulation process is concluded if the escape rate in the steady state does not change by more than $10 \%$ when the number of grid cells is doubled. It is noted that in the case of HEP $=15$ using 3200 grid cells, as much as $30 \%$ of the computing time can be saved by using grid continuation, a process using steady state results of simulations with a small number of grid points as initial conditions for the calculations using a large number of grid points.

Because these simulations of an isothermal atmosphere produce a good approximation of the steady state hydrodynamic escape rate and large-scale atmospheric structure, these validation

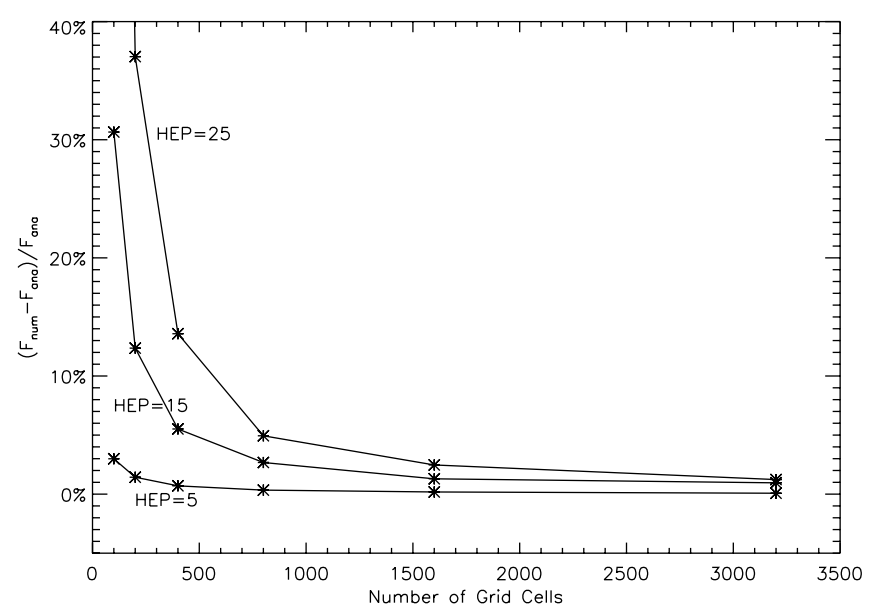

FIG. 3.-Analytical solutions of hydrodynamic escape from an isothermal atmosphere are approached when the number of grid cells is increased (first-order scheme). The vertical axis is the relative deviation of the escape flux in the numerical solution compared with that in the analytical solutions for different HEP values. 

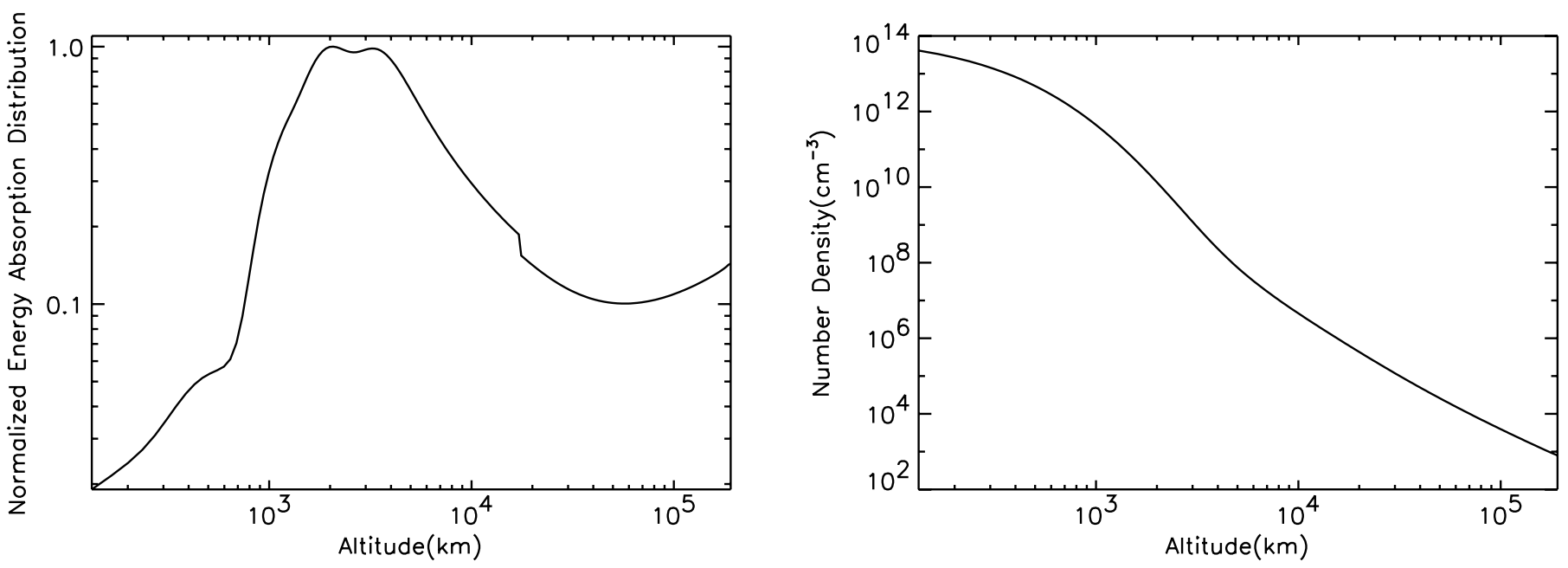

FIG. 4. $-\mathrm{H}_{2}$ number density distribution in an atmosphere of an Earth-like planet and the corresponding normalized energy absorption distribution, defined as the energy absorption rate (ergs s${ }^{-1}$ ) divided by the maximum energy absorption rate ( $\mathrm{ergs} \mathrm{s}^{-1}$ ).

results show that our numerical method is capable of accurately solving equation (1) for transonic flows.

\section{IMPLICATION OF THE SINGLE-LAYER HEATING ASSUMPTION AND OTHER ENERGY CONSIDERATIONS}

Watson et al. (1981) assumed that heating due to solar EUV radiation occurs only in a single layer. This assumption is not necessarily a good approximation, because the absorption cross section depends on wavelength. The unity optical depth level occurs at a different altitude for each wavelength. As a result, the energy absorption altitude distribution may have a complicated structure. Figure 4 shows the $\mathrm{H}_{2}$ number density distribution in an atmosphere of an Earth-like planet (obtained by simulation assuming single-layer heating and a homopause $\mathrm{H}_{2}$ density of $5 \times 10^{13} \mathrm{~cm}^{-3}$ ) and the corresponding normalized energy absorption distribution (calculation method described in a later part of this section). The spectrum of solar EUV radiation entering the top of the atmosphere is based on UARS SOLSTICE measurements from 119 to $420 \mathrm{~nm}$ and a 1994 rocket measurement from 0 to $119 \mathrm{~nm}$ (Woods \& Rottman 2002). The absorption cross section data for $\mathrm{H}_{2}$ gas are from Avakyan et al. (1998). Insignificant Rayleigh scattering is neglected. The same solar spectra and absorption cross section data are used for all simulations in this paper. Figure 4 shows that most of the energy is absorbed at altitudes above $1000 \mathrm{~km}$. The double-peaked feature between $10^{3}$ and $10^{4} \mathrm{~km}$ is caused by the wavelength dependence of the energy deposition (Chassefière 1996). The energy deposition levels off and then starts to increase at around $10^{5} \mathrm{~km}$ because of the geometric effect (Chassefière 1996) of the increasing size of the extended planetary atmosphere (see $\S 4$ ).

We first present results calculated with our numerical model using the single-layer heating assumption. The hydrogen molecule escape rates corresponding to different heating layer positions and energy deposition rates are calculated for the atmosphere of an Earth-like planet, with the lower boundary hydrogen density set to be $10^{14} \mathrm{~cm}^{-3}$ (Fig. 5). For an energy deposition rate of $0.15 \mathrm{ergs} \mathrm{cm}^{-2} \mathrm{~s}^{-1}$, a value equivalent to today's solar EUV radiation input into the Earth's atmosphere (Watson et al. 1981), the escape rate changes with varying heating layer altitudes. Since solar EUV radiation was much stronger in the early history of the Earth, a larger energy deposition rate is plausible (Walter $\&$ Barry 1991). For an energy deposition rate of $0.3 \mathrm{ergs} \mathrm{cm}^{-2}$ $\mathrm{s}^{-1}$, the escape rate is a much steeper function of the heating layer altitude than for an energy deposition rate of 0.15 ergs $\mathrm{cm}^{-2} \mathrm{~s}^{-1}$. Thus, the escape rates depend on the position of the heating layer as well as on the absolute amount of energy input. It is difficult for a model with a single heating layer to represent the dependence accurately.

All energy deposition calculations in the literature assume that solar radiation enters the top of the atmosphere isotropically. To account for the angular distribution of solar radiation, the mass path is usually calculated for an average solar zenith angle of $\sim 60^{\circ}$ (Kasting \& Pollack 1983; Chassefière 1996). Although this approach is a good approximation for a thin, plane-parallel atmosphere, it is not appropriate for a dense, extended atmosphere, in which radiative transfer in spherical geometry is needed. Because of the lack of spherical symmetry, the problem of radiative transfer in an extended spherical atmosphere is complicated, and easy analytical expressions are not available (Sobolev 1975). A two-dimensional energy deposition calculation method is developed to provide a more accurate approximation of the complicated radiative transfer processes in an extended spherical atmosphere.

As shown in Figure $6 a$, a solar radiation beam is absorbed (Beer's Law) in a layer with depth $\Delta s$, which is a function of the

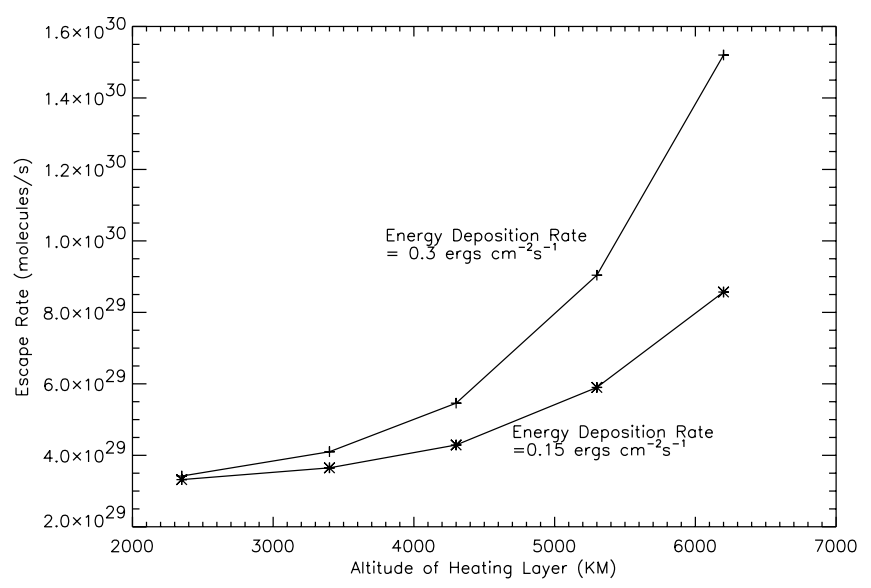

FIG. 5.-Nonlinear relations between escape rates and the position of the heating layer under the single-layer heating assumption of Watson et al. (1981). The relation depends on the energy deposition rate. 


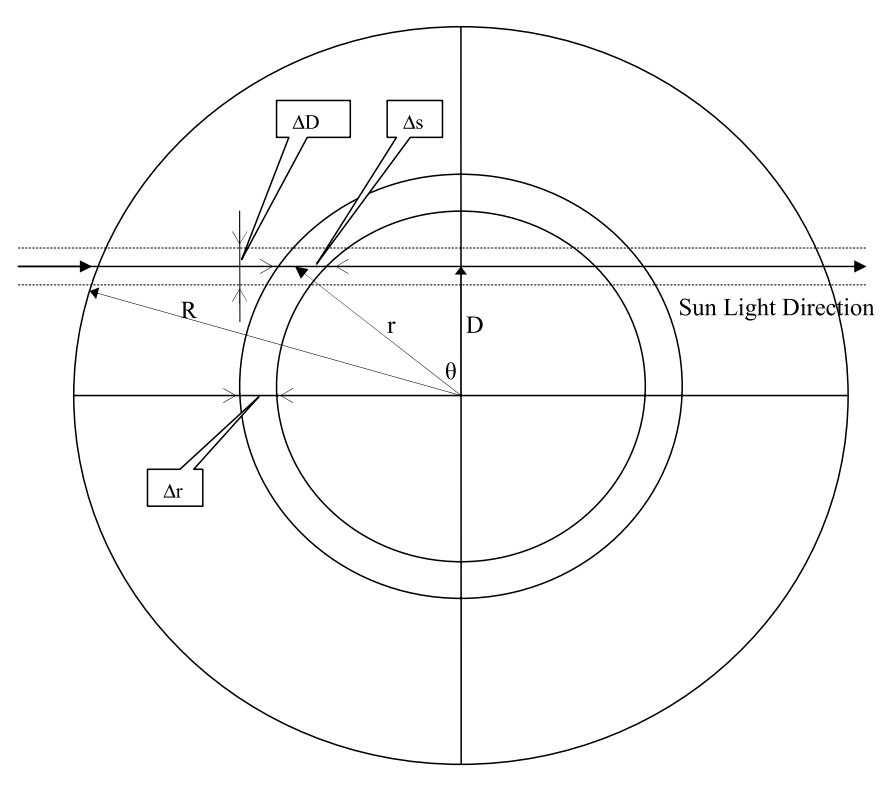

FIG. $6 a$

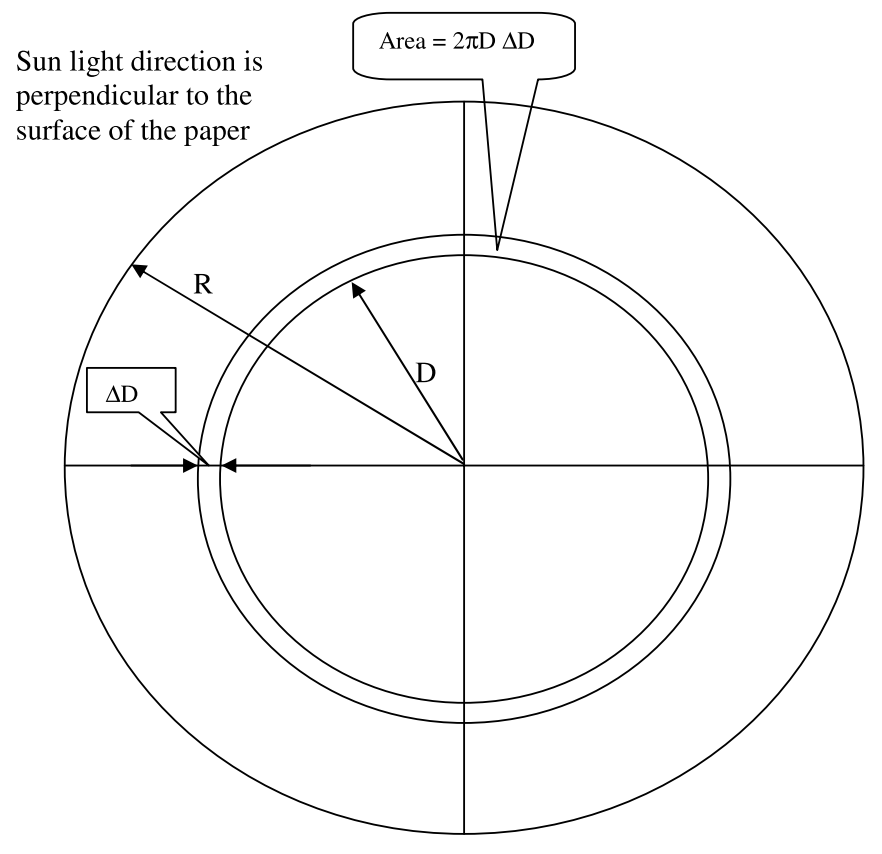

FIG. $6 b$

Fig. 6.-Schematic of the two-dimensional energy deposition calculation method. (a) Solar beam is in the plane of the figure. (b) Solar beam is perpendicular to the figure.

azimuth angle $\theta$ and the radial depth of the layer $\Delta r$. The energy deposition in a given layer along each path is then multiplied with the area of the ring, $2 \pi D \Delta D$ (Fig. $6 b$ ), to obtain the total energy deposited into the shell segment. Here $D$ is the distance from the planet center to the solar radiation beam. Note that for one shell with radius $r$, shell segments may have different $D$ values (from $D=0$ to $D=r$ ). When all shell segments belonging to the same shell are included, the total energy deposited into the shell is obtained. The total energy divided by the total volume of the shell gives the volume heating rate in the shell. For an atmosphere with an extremely low density, the volume heating rate should be nearly zero everywhere. For an atmosphere with an extremely high density (such as a solid body), all energy should be absorbed in the hemisphere facing the Sun, and the energy deposited into the surface layer should be $F \pi R^{2}$, where $F$ is the solar flux constant at the orbit of the planet and $R$ is the radius of the atmosphere. These two extreme limits are produced by the two-dimensional numerical model.

Not all energy absorbed by hydrogen molecules will be used to drive hydrodynamic escape flow. Some will be lost to space through thermal emission. Although the thermal emission process is not included in this paper, a heating efficiency $(\eta)$ is defined as the percentage of energy absorbed by molecules that is effectively converted into the kinetic energy of the molecules. In Watson et al. (1981), $\eta=15 \%$. We use the same value in $\S 4$, where we discuss transonic hydrodynamic escape from the atmosphere of an Earth-like planet as the first approximation. In $\S 5$ (application of the model to atmospheres of extrasolar planets) we discuss in more detail the importance of different heating efficiency values for the simulation results.

\section{HYDRODYNAMIC ESCAPE}

\section{FROM A HYDROGEN-DOMINATED ATMOSPHERE}

Our model and the energy calculation method are applied to the atmosphere of an Earth-like planet in which the thermosphere is composed only of hydrogen molecules. We set the simulation parameters to be similar to those used by Watson et al. (1981) so that simulation results can be compared with those of Watson. The lower boundary is fixed at approximately the altitude of the homopause $(\sim 100 \mathrm{~km})$, and the lower boundary temperature is fixed at $250 \mathrm{~K}(\mathrm{HEP}=42)$. The upper boundary is at an altitude of roughly $10^{5} \mathrm{~km}$. The solar EUV radiation flux is that of today $(\S 3)$. The hydrogen density at the lower boundary is treated as a free parameter, and four cases are summarized in Table 2. The total energy deposition rate is obtained by adding the energy deposited in each layer per unit time together. Figure 7 shows the change of escape rate with varying homopause hydrogen density. Figures 8, 9, and 10 show the steady state distributions of density, gas flow velocity, and temperature for the different cases in Table 2.

The density distributions in the steady state of hydrodynamic escape from a hydrogen-dominated atmosphere of an Earth-like planet are shown as solid curves in Figure 8. The density distributions deviate severely from those in hydrostatic equilibrium (dashed and dotted curves in Fig. 8). Therefore, the atmospheric structure cannot be described properly by the concept of the scale height (the altitude within which the density drops by a factor of $e$ ). Thus, the traditional definition of the exobase (the altitude at which the mean free path of particles is comparable to the scale height of the atmosphere) is not appropriate, and an equivalent definition of the exobase is the

TABLE 2

Escape Calculations in the Atmosphere of an Earth-like Planet

\begin{tabular}{|c|c|c|}
\hline $\begin{array}{c}\text { Homopause } \mathrm{H}_{2} \text { Number } \\
\text { Density } \\
\left(\mathrm{cm}^{-3}\right)\end{array}$ & $\begin{array}{c}\text { Escape Rate } \\
\left(10^{29} \mathrm{H}_{2} \mathrm{~s}^{-1}\right)\end{array}$ & $\begin{array}{c}\text { Total Energy Deposition } \\
\text { Rate } \\
\left(10^{16} \mathrm{ergs} \mathrm{s}^{-1}\right)\end{array}$ \\
\hline Case A: $5 \times 10^{11}$. & 0.20 & 7.5 \\
\hline Case B: $5 \times 10^{12}$. & 2.03 & 10.0 \\
\hline Case C: $5 \times 10^{13} \ldots$ & 5.04 & 15.3 \\
\hline Case D: $5 \times 10^{14}$ & 12.94 & 22.3 \\
\hline
\end{tabular}




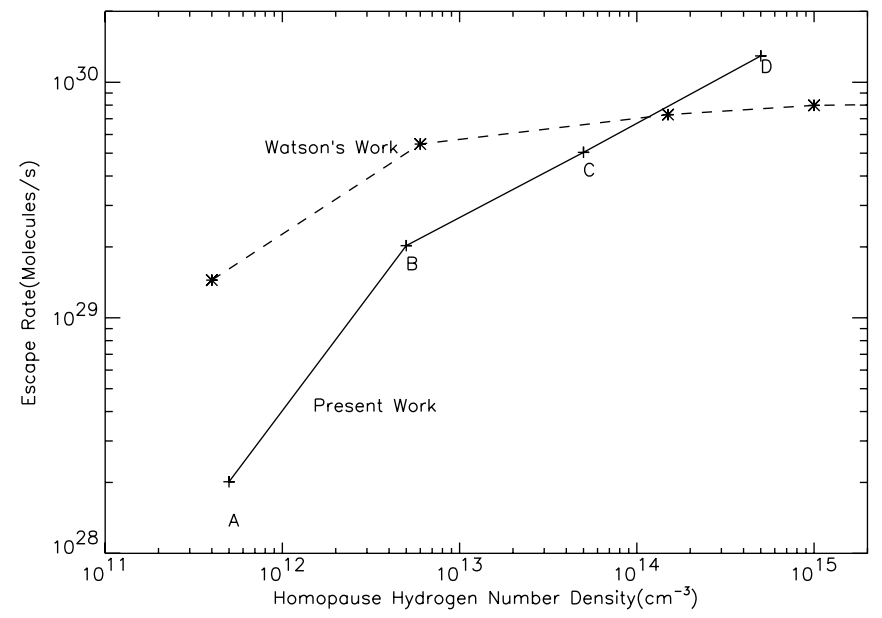

FIG. 7.-Escape rate calculation using our method and that of Watson et al. (1981) in a hydrogen-dominated atmosphere of an Earth-like planet.

altitude at which the probability of collisions with the particles above the altitude is unity, or $\int_{r^{\infty}}^{\infty} n(r) \sigma d r \sim 1$ with collisional cross section $\sigma=3.33 \times 10^{-15} \mathrm{~cm}^{2}$ (Hunten 1973). The asterisks in Figure 8 show the positions of the exobases.

Near the upper boundary, the gas flow velocities become supersonic except in cases of low hydrogen homopause density (Fig. 8). Because of the extended heating source, the gas flow velocities near the upper boundary in our simulation are greater than those in Watson et al. (1981). The dashed curve in Figure 9 shows roughly the velocity profile in case $\mathrm{C}$ of Watson et al. (1981). The general shapes of the density and velocity profiles are similar to the previous simulation results (Watson et al. 1981; Kasting \& Pollack 1983). In case A, the column density of hydrogen in the model is so small that the absorbed energy is not enough to support a supersonic escape flow. Similar subsonic hydrodynamic escape scenarios can be found in Kasting $\&$ Pollack (1983). Because of the decline of gravity with dis-

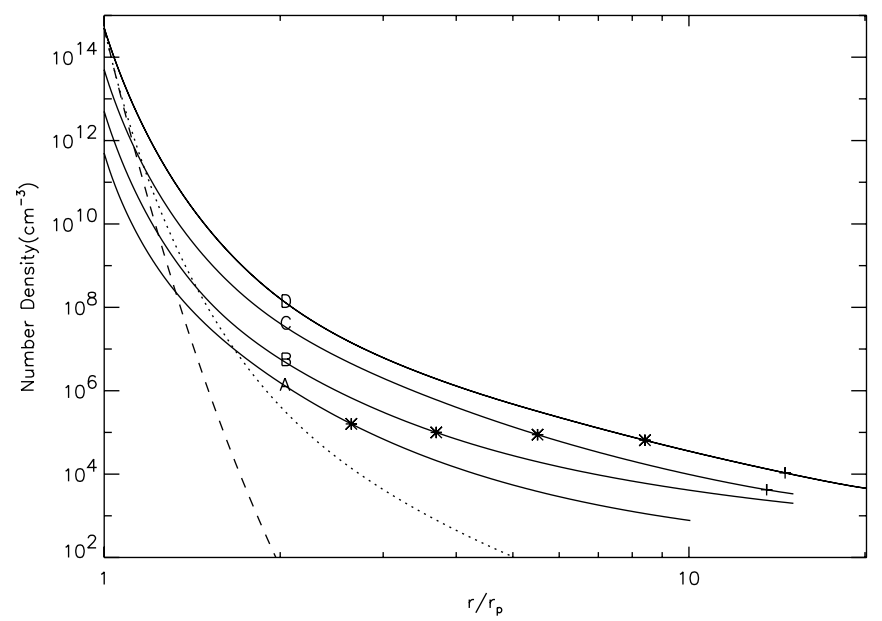

FIG. 8.- Solid curves show the density distributions in the steady state of hydrodynamic escape from a hydrogen-dominated atmosphere of an Earth-like planet. The dashed curve is the density distribution in isothermal $(250 \mathrm{~K})$ hydrostatic equilibrium with the same homopause hydrogen density as in case D. The dotted curve is the density distribution given both the homopause hydrogen density and the temperature profile in Fig. 10. Because of the severe deviations of the density distribution from those in hydrostatic equilibrium, the atmospheric structure cannot be described properly by the concept of the scale height (the altitude within which the density drops by a factor of $e$ ). A new definition of the exobase is employed. The positions of the exobases are shown by the asterisks. The transonic points are marked by the plus signs.

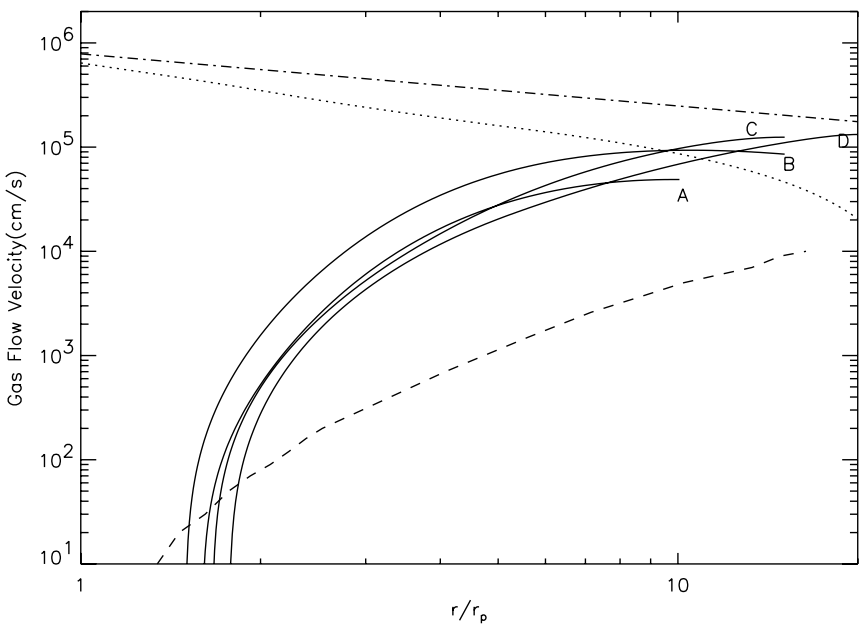

FIG. 9.-Velocity distributions in the steady state of hydrodynamic escape from a hydrogen-dominated atmosphere of an Earth-like planet. The dot-dashed curve shows the escape velocity from such an atmosphere. The dotted curve shows the difference of the thermal velocity and the escape velocity. The Watson et al. (1981) result is shown as the dashed curve. The transonic points are shown in Fig. 8.

tance, near the upper boundary the escape velocity (shown by the dot-dashed curve in Fig. 9) becomes comparable to the gas flow velocity. Because the escape velocity will continue to decrease and the gas flow velocity remains nearly constant beyond the upper boundary, it can be concluded that all materials in the escape flow will be lost from the planet, and the selection of the position of the upper boundary is justified. Moreover, the gas molecules have not only a flow velocity, but also a thermal velocity. The dotted curve illustrates the difference of the thermal and the escape velocity, which is exceeded by the flow beyond $\sim 9 R_{p}$, at which point half of the molecules will clearly escape.

The temperature (Fig. 10) varies with escape flow because the escape flow carries out energy and produces adiabatic cooling. A large escape rate results in a relatively low asymptotic temperature in the upper atmosphere (case D). The temperature profiles in the upper atmosphere in all cases asymptotically approach different constant values. The temperature becomes isothermal in the upper atmosphere, similar to the results of

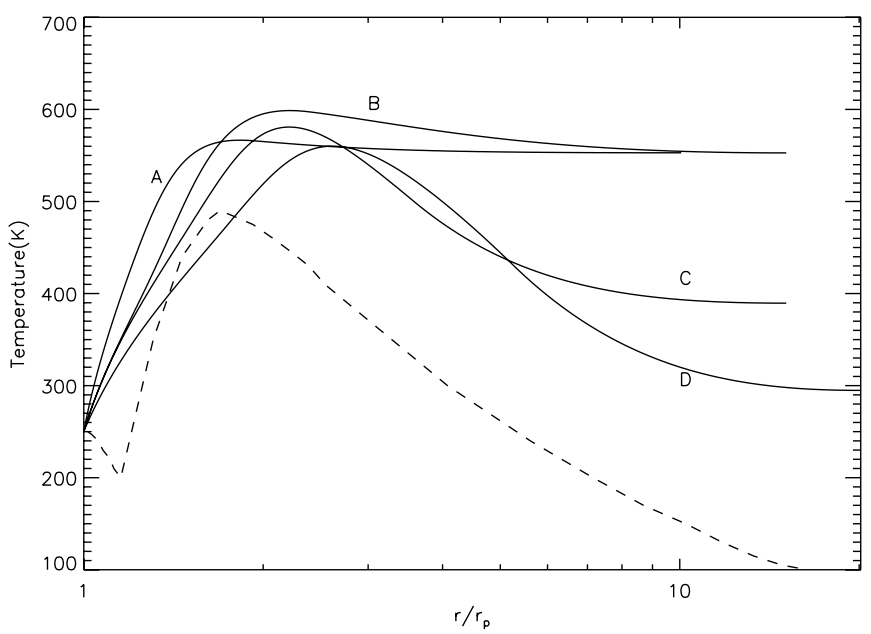

FIG. 10.-Temperature distributions in the steady state of hydrodynamic escape from a hydrogen-dominated atmosphere of an Earth-like planet. The dashed curve shows roughly the temperature profile of case $\mathrm{C}$ in Watson et al. (1981). 
Kasting \& Pollack (1983), and does not resemble the profiles in Watson et al. (1981). In Watson et al. (1981), a "cold trap" is formed in the lower part of the atmosphere when the escape flux is large, because the thermal conduction cannot compensate for the quick loss of energy accompanying the fast escape flow. In our simulations, because energy absorption occurs over a broad range of altitudes, the cold trap in Watson's results does not form. The dashed curve in Figure 10 shows roughly the temperature profile of case C in Watson et al. (1981).

In addition to the hydrogen homopause density, there are two effects related to the energy deposition and distribution that affect the escape rate. First, the total amount of EUV radiation absorbed is smaller in a low homopause density atmosphere than in a high homopause density atmosphere. Second, most of the energy absorption occurs at lower altitudes when the homopause hydrogen density is low, which causes a large temperature gradient near the lower boundary. With a large temperature gradient, energy is quickly lost through thermal conduction out of the lower boundary, which contributes to a weak escape flow. These nonlinear effects combined together produced smaller escape rates in our simulations for homopause hydrogen densities below $10^{14} \mathrm{~cm}^{-3}$ compared with Watson et al. (1981). The escape rates are higher in our simulations than in Watson's for large hydrogen abundances because more energy is absorbed in the extended atmosphere than Watson assumed.

\section{APPLICATIONS AND DISCUSSIONS}

We now apply our model to the hydrodynamic escape of hydrogen from an extrasolar planet. Almost all extrasolar planets discovered so far are giant planets with dense hydrogen-rich atmospheres orbiting close to their stars. Because of the close distances from their parent stars, the stellar radiation deposited in the atmosphere of such hot Jupiters can be so strong that the hydrodynamic escape of hydrogen can be stimulated.

A $15 \%$ reduction in the stellar Ly $\alpha$ emission line has been observed during the transit of extrasolar planet HD 209458b (Vidal-Madjar et al. 2003). Since the planet itself covers only approximately $1.5 \%$ of its parent star's surface during the transits (Vidal-Madjar et al. 2003), the observation suggests the existence of a dense hydrogen cloud extended to $\sim 4$ planetary radii. The formation of this hydrogen atom cloud requires efficient formation of hydrogen atoms from hydrogen molecules under high temperature $\left(10^{4}-10^{5} \mathrm{~K}\right)$ and efficient transport of hydrogen from the lower to the upper atmosphere (most likely through a hydrodynamic escape process). The observed Ly $\alpha$ reduction spectrum has a half-width of roughly $0.5 \AA$ (VidalMadjar et al. 2003), which suggests strong Doppler broadening of the Ly $\alpha$ emission line when it passes through the hydrogen cloud of the extrasolar planet HD 209458b. Recently, oxygen and carbon absorption has been observed during the transit of extrasolar planet HD 209458b (Vidal-Madjar et al. 2004). Vidal-Madjar et al. (2004) pointed out that explaining these new observations requires a high hydrogen density $\left(>10^{6} \mathrm{~cm}^{-3}\right)$ near the boundary of the Roche lobe and a gas flow velocity of at least $10 \mathrm{~km} \mathrm{~s}^{-1}$. (The Roche lobe is the volume surrounding an object within which material is gravitationally bound to it. The Roche lobe of HD 209458 b extends to $\sim 3.6$ Jupiter radii.)

It is complicated to understand the actual physical and chemical processes in the atmosphere of an extrasolar planet. One would need to understand the interaction of the planet with the stellar wind and to model the photochemistry in the atmosphere as well as the detailed radiative transfer. Given our limited knowledge of these objects, it is beneficial to obtain some basic information on the radial structure (density, velocity, tempera- ture) of the upper atmosphere of extrasolar planets through onedimensional simulations of hydrogen hydrodynamic escape from these planets. Our simulation results are compared with the observations by Vidal-Madjar et al. (2003, 2004).

The parent star of the transiting extrasolar planet HD 209458b is close to solar type (Vidal-Madjar et al. 2003). To apply the hydrodynamic model to extrasolar planets, we assume that the stellar EUV radiation is the only energy source driving the escape flow and that the radiation spectrum is the same as that of the Sun. The lower and upper boundaries for the simulation presented here are at 1 planetary radius $\left(R_{p}=1.35 R_{\mathrm{J}}\right.$; Sudarsky et al. 2003) and $10 R_{p}$, respectively. The mass of the planet is 0.69 Jupiter mass. At the upper boundary, the escape flow becomes supersonic, so no boundary condition is imposed. It is suggested that the atmosphere temperature at $1 R_{p}$ is approximately $750 \mathrm{~K}$ (Goukenleuque et al. 2000). For hydrogen molecule escape, this temperature results in HEP $=187$. The heating efficiency $(\eta)$ in planetary atmospheres is poorly constrained; for example, $\eta$ in the upper atmosphere of Jupiter is $\sim 63 \%$ (Waite et al. 1983), while $\eta$ in a hydrogen-rich atmosphere of early Earth is $~ 30 \%$ (Watson et al. 1981) and $\eta=15 \%$ in a water-rich early Venus (Kasting \& Pollack 1983). Three heating efficiency values $(10 \%$, $30 \%$, and $60 \%$ ) are for our sensitivity test simulations. Our simulations are divided into two categories (pure hydrogen molecule and pure hydrogen atom), because there can be only one gas component in the current version of our hydrodynamic escape model. The transformation from hydrogen molecules to hydrogen atoms is not accounted for explicitly, but the results should be between the two extreme cases.

Simulations show that the position of the exobase in the extended atmosphere of HD $209458 \mathrm{~b}$ is close to $10 R_{p}$ (Fig. 11). The velocity of the escape flow is supersonic near the upper boundary (the sound speed is $\sim 10 \mathrm{~km} \mathrm{~s}^{-1}$ at $10^{4} \mathrm{~K}$ ) and exceeds the escape velocity (light blue curve in Fig. 11) at $\sim 3 R_{p}$, which validates the supersonic upper boundary settings. The high temperature $\left(10^{4}-10^{5} \mathrm{~K}\right)$ in the upper atmosphere is adequate for efficient formation of hydrogen atoms from hydrogen molecules. A hydrogen density of more than $10^{6} \mathrm{~cm}^{-3}$ near the Roche lobe $\left(\sim 3.6 R_{\mathrm{J}}\right)$ and a large gas flow velocity $\left(\sim 10 \mathrm{~km} \mathrm{~s}^{-1}\right)$ is present in all simulation results. These features are consistent with the Ly $\alpha$ emission-line observations (Vidal-Madjar et al. 2003) and the recent $\mathrm{O}_{\mathrm{I}}$ and $\mathrm{C}$ II observations (Vidal-Madjar et al. 2004). Table 3 compares the observation results directly with the simulation results.

The observed 15\% Ly $\alpha$ emission-line reduction during the transit may be produced by a hydrogen cloud opaque to Ly $\alpha$ (optical depth $\sim 1$ ) and with a radius of $\sim 4 R_{p}$. The same amount of reduction can be created if the hydrogen cloud covers the whole star but has an average optical depth of $\sim 0.15$. Without spatial resolution in the observations, it is impossible to distinguish the possible scenarios. On the other hand, the Ly $\alpha$ emission-line profile observed during the transit of HD 209458b is a better constraint on the structure of the hydrogen cloud than the emissionline reduction is.

Ly $\alpha$ emission is produced by recombination of hydrogen ions (Gonzalez Delgado et al. 1998). When passing through an astrophysical medium, $\operatorname{Ly} \alpha$ photons can experience resonant scattering by hydrogen atoms in the ground state (Neufeld 1990). In addition to scattering, Ly $\alpha$ photons can be absorbed by dust or molecules. They can also be lost through collisional de-excitation of a scattering hydrogen atom when it is in the excited state or be converted into UV-fluorescent photons in warm molecular gas (Neufeld 1990). The resonant scattering by neutral hydrogen atoms can greatly increase the optical path length of $\operatorname{Ly} \alpha$ photons 

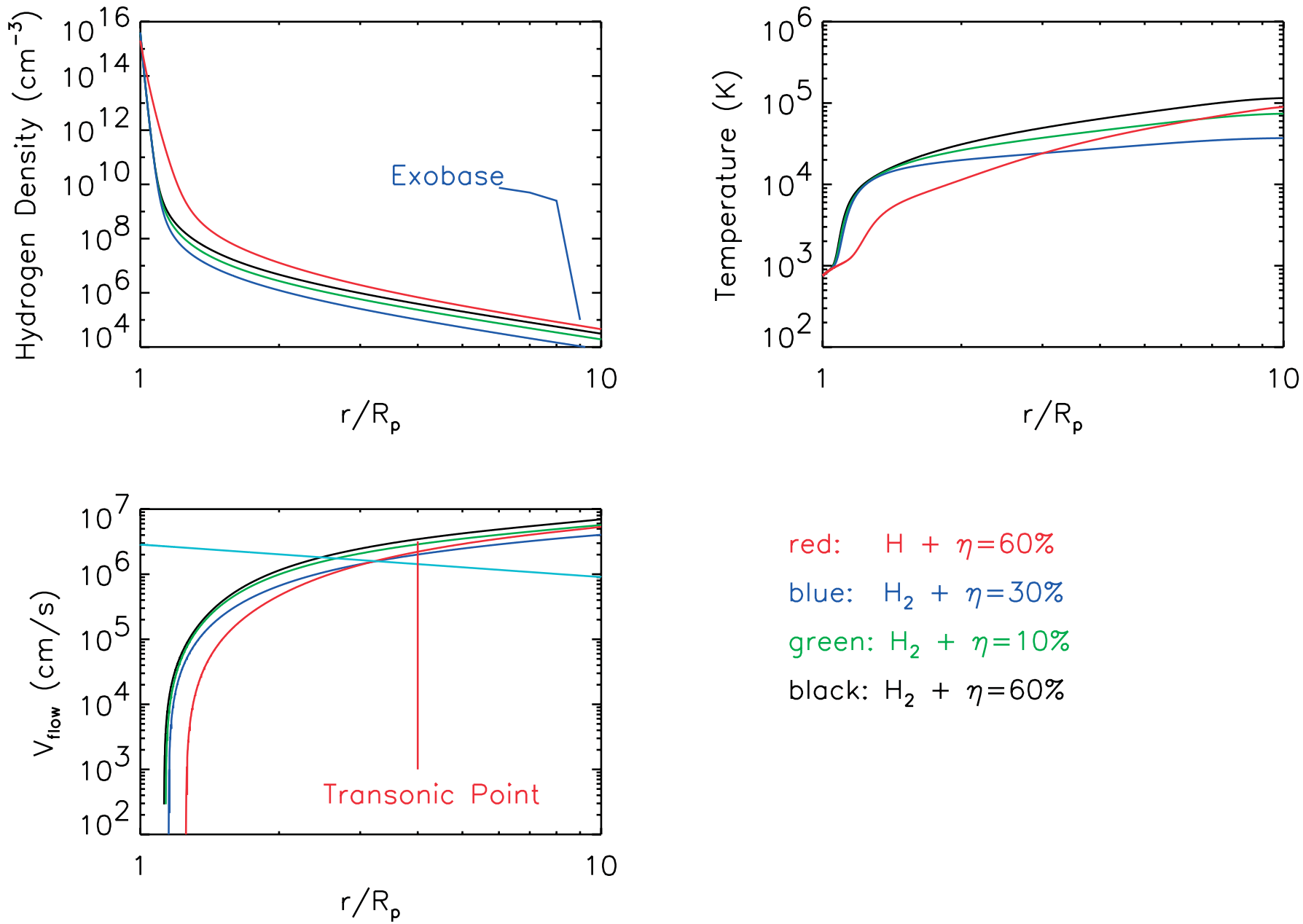

$$
\begin{array}{ll}
\text { red: } & H+\eta=60 \% \\
\text { blue: } & \mathrm{H}_{2}+\eta=30 \% \\
\text { green: } \mathrm{H}_{2}+\eta=10 \% \\
\text { black: } \mathrm{H}_{2}+\eta=60 \%
\end{array}
$$

FIG. 11. - Simulation results of hydrodynamic escape from HD 209458b. Here $\eta$ is the heating efficiency. The position at which the gas flow becomes transonic and the position of the exobase are marked. The light blue curve represents the escape velocity from the extrasolar planet.

and the probability that Ly $\alpha$ photons will be absorbed by dust (Kunth et al. 1998). Asymmetric profiles of the Ly $\alpha$ emission line (P Cygni type profile) have been observed from starburst galaxies (Ahn et al. 2001). The profile can be explained by the expanding neutral gas surrounding the Ly $\alpha$ source (Gonzalez Delgado et al. 1998). Studies suggest that the emergent $\mathrm{Ly} \alpha$ profile is sensitive to various factors, including the kinematics, the scattering optical depth, and the dust content of the neutral gas (Ahn et al. 2001; Gonzalez Delgado et al. 1998; Kunth et al. 1998).

Without considering the contribution to line broadening from the escaping gas velocity, we find that the observed Ly $\alpha$ emissionline profile during the transit of the extrasolar planet cannot be explained. The scattering optical depth of $\operatorname{Ly} \alpha$ photons follows the Voigt function $\tau_{x}=\tau_{0} H(x, a)$, where $a=4.71 \times 10^{-4} T_{4}^{-1 / 2}$ is the Voigt parameter, $T_{4}$ is the temperature of the medium in units of $10^{4} \mathrm{~K}$, and $x \equiv \Delta \nu / \Delta \nu_{\mathrm{D}}=\left(\nu-\nu_{0}\right) / \Delta \nu_{\mathrm{D}}$ is a dimensionless parameter. Here $\Delta \nu_{\mathrm{D}} \equiv \nu_{0}\left(V_{\text {th }} / c\right)$ is the Doppler shift, where $V_{\text {th }}$ is the thermal speed of the scattering hydrogen cloud. The line center optical depth is $\tau_{0}=1.41 \times 10^{-13} N\left(\mathrm{H}_{0}\right) T_{4}^{-1 / 2} \mathrm{~cm}^{2}$, where $N\left(\mathrm{H}_{0}\right)$ is the hydrogen atom column density (Ahn et al. 2001). Using the hydrogen densities at $4 R_{p}$ from the simulation results, in order to have unity optical depth at a $0.5 \AA$ deviation from the $\operatorname{Ly} \alpha$ line center, the temperature must be at least on the order of $10^{5} \mathrm{~K}$. This temperature is about a factor of 5 greater than the values obtained in simulations at $4 R_{p}$. Given the temperatures in our simulation results, the line broadening is only $0.2-0.3 \AA$, which is smaller than the observed $0.5 \AA$ line width (Vidal-Madjar et al. 2003).

However, by taking into consideration the high gas flow velocity $\left(>10 \mathrm{~km} \mathrm{~s}^{-1}\right.$ above $\left.3 R_{p}\right)$, the strong Doppler broadening of

\begin{tabular}{|c|c|c|c|}
\hline Requirements and Simulations & $\begin{array}{c}\text { Temperature } \\
\text { (K) }\end{array}$ & $\begin{array}{l}\text { Gas Flow Velocity } \\
\left(\mathrm{km} \mathrm{s}^{-1}\right)\end{array}$ & $\begin{array}{l}\text { Hydrogen Density } \\
\qquad\left(\mathrm{cm}^{-3}\right)\end{array}$ \\
\hline Observation requirements .......................... & $10^{4}-10^{5}$ & $\geq 10$ & $>10^{6}$ \\
\hline 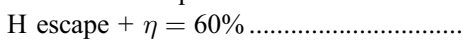 & $9.65 \times 10^{4}$ & 13.9 & $1.93 \times 10^{6}$ \\
\hline 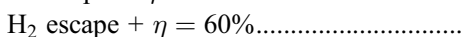 & $4.95 \times 10^{4}$ & 24.7 & $0.97 \times 10^{6}$ \\
\hline 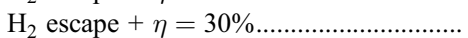 & $3.74 \times 10^{4}$ & 20.8 & $0.58 \times 10^{6}$ \\
\hline 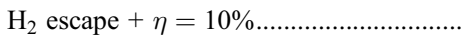 & $2.42 \times 10^{4}$ & 14.3 & $0.26 \times 10^{6}$ \\
\hline
\end{tabular}

TABLE 3

Direct Comparison between Parameters Near the Roche Lobe $\left(\sim 3.6 R_{\mathrm{J}}\right)$ in the Simulation Results AND the OBSERVATION Requirements 

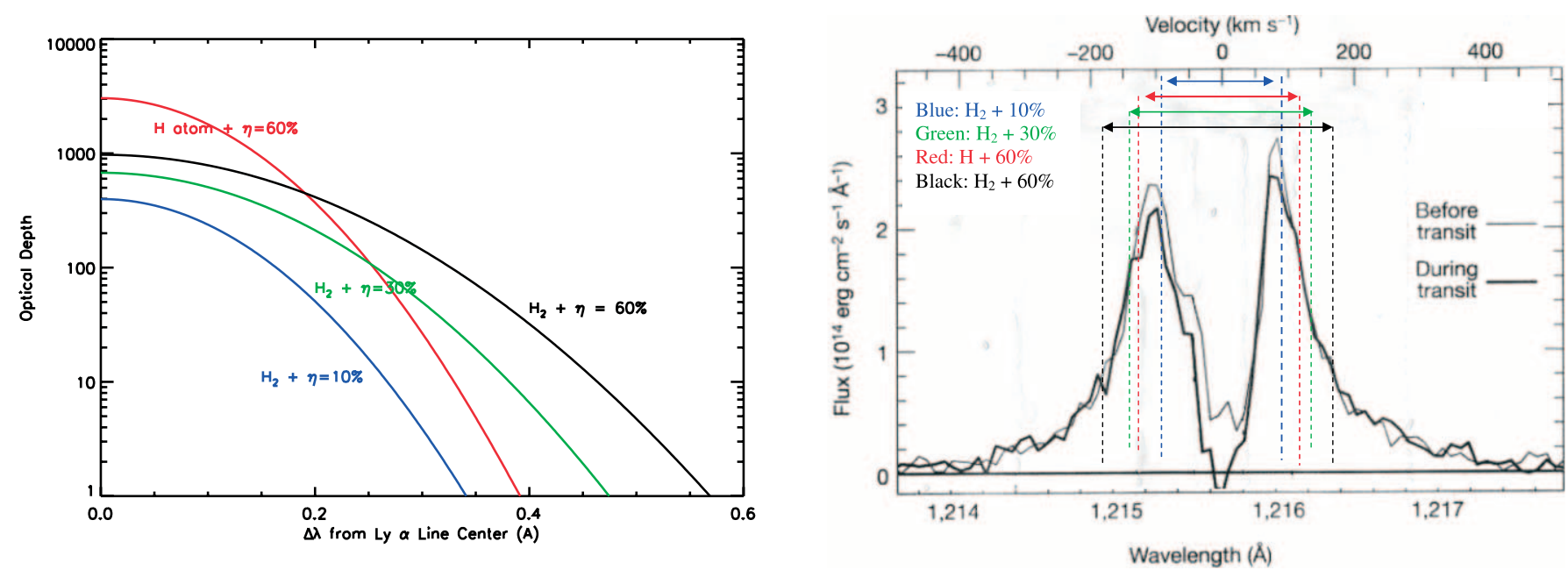

Fig. 12.-Combined effect of thermal broadening and broadening due to gas flow velocity in the extended hydrogen cloud of extrasolar planet HD 209458b. The parameters used in the calculations are at $4 R_{p}$ in the simulation results (shown in Fig. 11). Left: optical depth as a function of the wavelength. Right: Ly $\alpha$ reduction line width calculation compared with the observations (Vidal-Madjar et al. 2003).

the Ly $\alpha$ emission-line profile observed during the transit can be well explained. Unlike the asymmetric profile caused by the expanding neutral gas in the starburst galaxies, the line shift caused by the escape flow from extrasolar planet HD 209458b should be roughly symmetric. Ly $\alpha$ photons have to go through the part of the hydrogen cloud with gas flowing toward the star and the part with gas flowing away from the star. Because of the radiation pressure and gravitational force from the star, the hydrogen cloud structure should have a certain degree of asymmetry (Vidal-Madjar et al. 2004). As a first approximation, we ignore these effects here. Thus, the total Doppler shift can be expressed as $\Delta \nu_{\mathrm{D}} \equiv \nu_{0}\left(V_{\text {th }}+V_{\text {flow }}\right) / c$, where $V_{\text {flow }}$ is the gas flow velocity. With the correctly defined Doppler shift, Voigt parameter, and line center optical depth, Figure 12 shows the combined effect of the thermal broadening and line shift due to high gas flow velocity in the extended hydrogen cloud of extrasolar planet HD 209458b. Parameters used in the calculations are at $4 R_{p}$ in the simulation results (Fig. 11). For the $\eta=30 \%$ ( $\eta$ is the heating efficiency) and $\eta=60 \%$ cases, unity optical depth can be reached at a broadened wavelength $\geq 0.5 \AA$ (Table 4), a good agreement with the Ly $\alpha$ observations (Vidal-Madjar et al. 2003).

Figure 13 shows that the hydrogen escape rates obtained from our simulations are in good agreement with the escape rate from the atmosphere of HD 209458b inferred by Vidal-Madjar et al. (2003). $\mathrm{H}$ atom escape leads to an escape rate roughly a factor of 2 higher than we calculate for $\mathrm{H}_{2}$ escape. It has been suggested that radiative cooling processes, especially those due to $\mathrm{H}_{3}{ }^{+}$, are important in the upper atmosphere of giant planets

TABLE 4

Wavelength Deviation from the Ly $\alpha$ Line Center

\begin{tabular}{|c|c|c|c|}
\hline Simulations & $\begin{array}{c}\tau=10 \\
(\AA)\end{array}$ & $\begin{array}{c}\tau=1 \\
(\AA)\end{array}$ & $\begin{array}{c}\tau=0.1 \\
(\AA)\end{array}$ \\
\hline $\mathrm{H}_{2}$ and $10 \%$ & 0.28 & 0.38 & 0.46 \\
\hline $\mathrm{H}_{2}$ and $30 \%$ & 0.42 & 0.55 & 0.65 \\
\hline $\mathrm{H}_{2}$ and $60 \%$ & 0.52 & 0.69 & 0.79 \\
\hline $\mathrm{H}$ and $60 \% \ldots \ldots \ldots \ldots \ldots \ldots \ldots \ldots \ldots$ & 0.41 & 0.50 & 0.58 \\
\hline
\end{tabular}

Note.-Escaping gas flow velocity and the thermal velocity both contribute to the broadening. Parameters for calculations are taken from $4 R_{p}$ in the simulation results (Fig. 11).
(Waite et al. 1997; Stallard et al. 2002). A 30\% reduction of the escape rate is obtained from a simulation assuming that the cooling function is $80 \%$ of the total heating function in the region in which most of the input energy is absorbed. It is noted that Jeans escape of hydrogen (which can be calculated from the atmospheric structure of the simulation results) is always an order of magnitude smaller than the hydrodynamic escape rates, which means that hydrodynamic escape is the dominant escape mechanism in the atmosphere of HD 209458b.

Lammer et al. (2003) calculated that the hydrogen escape rate from HD $209458 \mathrm{~b}$ is on the order of $10^{12} \mathrm{~g} \mathrm{~s}^{-1}$ by applying the Watson et al. (1981) approach. On the basis of the Watson approach, recently Baraffe et al. (2004) defined a "critical mass" as the mass below which the mass-loss timescale through

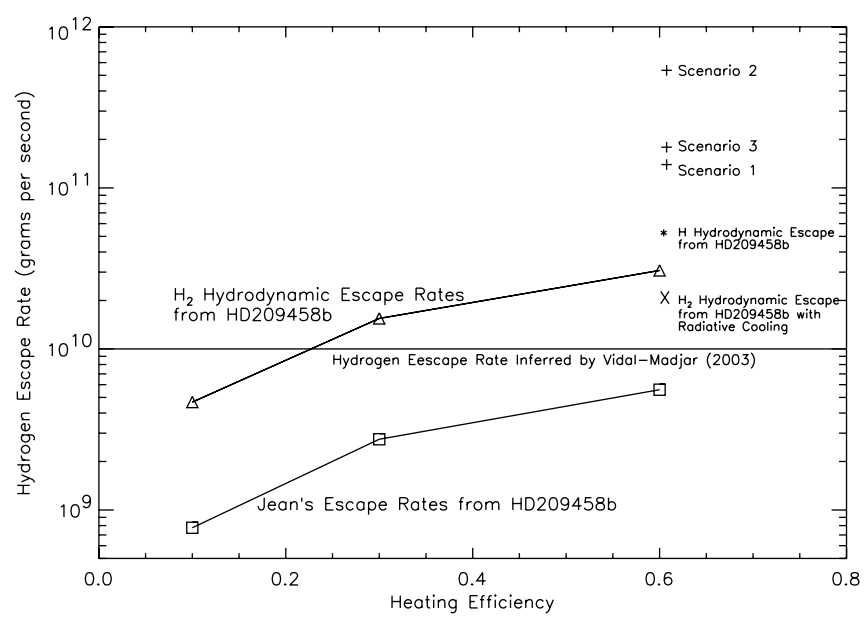

FIg. 13.-Escape rate calculations for extrasolar planets. The horizontal line is the escape rate $\left(10^{10} \mathrm{~g} \mathrm{~s}^{-1}\right)$ inferred in Vidal-Madjar et al. (2003). The upper curve is the $\mathrm{H}_{2}$ hydrodynamic escape rate under three assumed heating efficiencies. The lower curve represents the Jeans escape rates corresponding to the exobase temperatures in the calculations. Several special cases are computed for a heating efficiency of $60 \%$. If the escaping particles are hydrogen atoms instead of hydrogen molecules, the escape rate can be about a factor of 2 higher than our calculations for $\mathrm{H}_{2}$ escape. When radiative cooling is considered using a parameterization method, the escape rate can be reduced by about $30 \%$. Also shown in this graph are the hydrogen escape rates for three lower mass hypothetical extrasolar planets, as described in the text. 
atmospheric escape becomes $1 / 10$ of the Kelvin-Helmholtz timescale $\left(t_{\mathrm{K}-\mathrm{H}}=2 \mathrm{Gm}^{2} / R L\right)$ after 5 billion yr of evolution. The critical mass of extrasolar planets at the orbit of $\sim 0.05 \mathrm{AU}$ is proposed to be 1.5 Jupiter mass. As discussed in $\S 4$, the simplified energy treatment in Watson's approach may cause large errors in the estimate of the escape rate. The greatest hydrogen escape rate from our simulations for HD 209458b is about $6 \times 10^{10} \mathrm{~g} \mathrm{~s}^{-1}(60 \%$ heating efficiency and $\mathrm{H}$ atom escape). This result is very close to the hydrogen escape rate inferred from the Ly $\alpha$ observations (Vidal-Madjar et al. 2003). Our simulations show that the transiting extrasolar planet HD 209458b will lose only $1 \%$ of its mass in 6 billion yr and that its lifetime against mass loss through escape is on the order of $10^{11} \mathrm{yr}$. Lecavelier des Etangs et al. (2004) calculated the Jeans escape rate of hydrogen from extrasolar planets by considering the influence of tidal forces of the parent star on the position of the exobase. Their estimate of the lifetime of HD $209458 \mathrm{~b}$ is $10^{10}-10^{11} \mathrm{yr}$, similar to our estimates. Our simulation results suggest that the extrasolar planet HD 209458b can remain stable while satisfying all the observations (Vidal-Madjar et al. 2003, 2004) and that the proposed critical mass (1.5 Jupiter mass) for extrasolar planets near $0.05 \mathrm{AU}$ is an overestimation.

Although the transiting extrasolar planet HD 209458b is stable, a less massive extrasolar planet at the same orbit may experience much faster evaporation. By assuming the same lower boundary temperature, orbital distance from the parent star, and planetary mean density as HD 209458b, we simulated the hydrogen hydrodynamic escape from hypothetical smaller extrasolar planets. A planet with $1 / 8$ of the mass of HD 209458b (scenario 1 in Fig. 13) can lose $10 \%$ of its mass in 3.7 billion yr, but a planet with $1 / 27$ of the mass of HD 209458 b (scenario 2 in Fig. 13) can lose $10 \%$ of its mass within only about 0.28 billion yr. It is noted that in the calculations above we assumed the same mean density for hypothetical extrasolar planets with masses comparable to those of Uranus and Neptune, respectively. Because the mean density of a pure hydrogen planet changes strongly with its mass, a more complete study involving simulation of the planet's interior is needed to better determine the hydrogen hydrodynamic escape from small extrasolar planets. However, the numbers calculated here give an interesting first indication.

Simulation shows that through hydrodynamic escape of hydrogen, a planet at the orbit of Mercury $(0.4 \mathrm{AU})$ and with 0.5 Uranus mass (scenario 3 in Fig. 13) may lose about $10 \%$ of its mass within 850 million yr if the solar EUV radiation is 10 times the present level. Such a planet, if it existed, may lose all its atmosphere and expose its inside core to space. Our calculations and more systematic future studies may provide an indication on how Mercury may have evolved during the early days of the solar system.

Escape rates as large as $\sim 5 \times 10^{11} \mathrm{~g} \mathrm{~s}^{-1}$ can be achieved for a hypothetical extrasolar planet with the same characteristics as HD 209458b but at an orbit of 0.01 AU or if the stellar EUV radiation level is 25 times the present solar EUV level. This result is obtained without considering the possible increase of the bottom-level temperature under a more intense solar radiation level. The real hydrodynamic escape could be more dramatic. On the other hand, the close distance between the planet and the star means a higher ionization rate. A possible strong magnetic field of the planet may be effective in hindering the hydrodynamic escape of hydrogen. In addition to the magnetic field, at the short distance from the star the stellar gravitational force and radiation pressure will play more important roles in the dynamics of the extrasolar planet's atmosphere. All these effects can only be addressed by future study.
Without considering the gravitational disruption of extrasolar planetary atmospheres, a theoretical limit on the orbital distance of discoverable extrasolar planets can be established by studying the hydrogen hydrodynamic escape from the atmosphere of extrasolar planets close to their stars. This limit depends on the properties of the planet (mass, size) as well as the properties of the central star (EUV flux) and will be investigated in a future systematic study, which should include both considerations of the interior state and a more accurate radiative heating/cooling treatment. Fast escape of hydrogen may trigger a quick loss of volatiles $(\mathrm{C}, \mathrm{O}$, etc. $)$ from the planets and could produce volatilepoor planets. The impact of fast hydrodynamic escape of hydrogen on the evolutionary paths of small extrasolar planets is an interesting theoretical research topic.

\section{CONCLUSION}

In this paper a robust numerical model is developed to study transonic hydrodynamic escape from planetary atmospheres. The model is validated against analytical solutions of escape from an isothermal atmosphere. In previous studies of hydrodynamic escape, heating was assumed to occur in a single layer (Watson et al. 1981). In this paper we show that the single-layer heating assumption and other simple energy calculation methods in previous works are not sufficiently accurate for calculations of hydrodynamic escape from a hydrogen-rich planetary atmosphere.

A two-dimensional numerical model is developed to calculate the deposition of solar EUV radiation in an extended atmosphere. When compared with previous simulation results, our escape rate is higher than that of Watson et al. (1981) for large hydrogen abundances because of the greater total amount of energy absorbed in the extended atmosphere. Our escape rate is lower than Watson's for small hydrogen abundances because of the combination of a smaller amount of energy absorbed in the atmosphere and a greater loss of energy by thermal conduction at the lower boundary.

The numerical model, when applied to the atmospheres of extrasolar planets, provides profiles of density, velocity, and temperature in the steady state of hydrogen hydrodynamic escape flows. The resonant scattering of $\operatorname{Ly} \alpha$ photons, the Ly $\alpha$ resonance line profile, and the $0.5 \AA$ line width (Vidal-Madjar et al. 2003) are explained for the stellar transit of extrasolar planet HD 209458b. The hydrogen density and velocity required by the recent oxygen and carbon absorption observations (VidalMadjar et al. 2004) of extrasolar planet HD 209458b can be satisfied by our simulations. The good agreement between the simulation results and the observations suggests that hydrogen hydrodynamic escape is an ongoing process on extrasolar planet HD $209458 b$ and that the hydrogen cloud surrounding the extrasolar planet as a result of the hydrodynamic escape is the cause of the Ly $\alpha$ observation.

The greatest hydrogen escape rate from our simulations for HD $209458 \mathrm{~b}$ is about $6 \times 10^{10} \mathrm{~g} \mathrm{~s}^{-1}$. Under this escape rate, the planet will lose only $1 \%$ of its mass in 6 billion yr, which suggests that the extrasolar planet HD 209458b is stable under hydrodynamic escape. A planet with $1 / 27$ of the mass of HD 209458 b in the orbit of HD 209458 b may lose $10 \%$ of its mass within only about 0.28 billion yr. Hydrogen hydrodynamic escape from small extrasolar planets can significantly influence their long-term evolution. Simulation shows that through hydrodynamic escape of hydrogen, a planet at the orbit of Mercury ( $0.4 \mathrm{AU})$ and with 0.5 Uranus mass may lose about $10 \%$ of its mass within 850 million yr if the solar EUV radiation is 10 times the present level. This calculation provides an indication 
of how Mercury may have evolved during the early days of the solar system.

This study was supported by the NASA Institute of Astrobiology. We thank T. Woods, J. H. Waite, J. Ajello, L. Paxton,
W. Pryor, and Bill McClintock for providing valuable information on the solar EUV radiation spectrum and hydrogen absorption cross section data. We thank P. Maloney for valuable discussion on the Ly $\alpha$ emission line. We also thank J. F. Kasting and R. Yelle for valuable discussions on hydrodynamic escape from planetary atmospheres.
Ahn, S.-H., Lee, H.-W., \& Lee, H. M. 2001, ApJ, 554, 604

Avakyan, S. V., et al. 1998, Collision Processes and Excitation of UV Emission from Planetary Atmospheric Gases (Amsterdam: Gordon \& Breach)

Baraffe, I., Selsis, F., Chabrier, G., Barman, T. S., Allard, F., Hauschildt, P. H., \& Lammer, H. 2004, A\&A, 419, L13

Chassefière, E. 1996, J. Geophys. Res., 101(E11), 26039

De Sterck, H. 1999, Ph.D. thesis, Katholieke Univ. Leuven

De Sterck, H., Csík, A., Abeele, D. V., Poedts, S., \& Deconinck, H. 2001, J. Comput. Phys., 166, 28

Fox, J. L., \& Dalgarno, A. 1979, J. Geophys. Res., 84, 7315

Gonzalez Delgado, R. M., Leitherer, C., Heckman, T., Lowenthal, J. D., Ferguson, H. C., \& Robert, C. 1998, ApJ, 495, 698

Goukenleuque, C., Bézard, B., Joguet, B., Lellouch, E., \& Freedman, R. 2000, Icarus, 143,308

Hunten, D. M. 1973, J. Atmos. Sci., 30, 1481 1990, Icarus, 85,1

Hunten, D. M., Pepin, R. O., \& Walker, J. C. G. 1987, Icarus, 69, 532

Kasting, J. F., \& Pollack, J. B. 1983, Icarus, 53, 479

Keppens, R., \& Goedbloed, J. P. 1999, A\&A, 343, 251

Krasnopolsky, V. A. 1999, J. Geophys. Res., 104(E3), 5955

Kunth, D., Mas-Hesse, J. M., Terlevich, E., Terlevich, R., Lequeux, J., \& Fall, S. M. 1998, A\&A, 334, 11

Lammer, H., Selsis, F., Ribas, I., Guinan, E. F., Bauer, S. J., \& Weiss, W. W. 2003, ApJ, 598, L121

Lecavelier des Etangs, A., Vidal-Madjar, A., McConnell, J. C., \& Hébrard, G. 2004, A\&A, 418, L1

\section{REFERENCES}

Neufeld, D. A. 1990, ApJ, 350, 216

Parker, E. N. 1964, ApJ, 139, 72

Pavlov, A. A., \& Kasting, J. F. 2002, Astrobiology, 2, 27

Pepin, R. O. 1991, Icarus, 92, 2

Shizgal, B. D., \& Arkos, G. G. 1996, Rev. Geophys., 34, 483

Sobolev, V. V. 1975, Light Scattering in Planetary Atmospheres (Oxford: Pergamon)

Stallard, T., Miller, S., Millward, G., \& Joseph, R. D. 2002, Icarus, 156, 498

Sudarsky, D., Burrows, A., \& Hubeny, I. 2003, ApJ, 588, 1121

Toro, E. F. 1999, Riemann Solvers and Numerical Methods for Fluid Dynamics: A Practical Introduction (Berlin: Springer)

Vidal-Madjar, A., Lecavelier des Etangs, A., Désert, J.-M., Ballester, G. E., Ferlet, R., Hébrard, G., \& Mayor, M. 2003, Nature, 422, 143

Vidal-Madjar, A., et al. 2004, ApJ, 604, L69

Waite, J. H., Cravens, T. E., Kozyra, J., Nagy, A. F., Atreya, S. K., \& Chen, R. H. 1983, J. Geophys. Res., 88, 6143

Waite, J. H., Gladstone, G. R., Lewis, W. S., Drossart, P., Cravens, T. E., Maurellis, A. N., Mauk, B. H., \& Miller, S. 1997, Science, 276, 104

Walter, F. M., \& Barry, D. C. 1991, in The Sun in Time, ed. C. P. Sonett, M. S. Giampapa, \& M. S. Matthews (Tucson: Univ. Arizona Press), 633

Watson, A. J., Donahue, T. M., \& Walker, J. C. G. 1981, Icarus, 48, 150

Woods, T., \& Rottman, G. 2002, in Atomospheres in the Solar System: Comparative Aeronomy, ed. M. Mendillo, A. Nagy, \& J. H. Waite (Geophys. Monogr. 130; Washington: AGU), 221 